\begin{tabular}{ccc}
\hline International Journal of Engineering \& Technology, 7 (4.15) (2018) 315-317 & International Journal of Engineering \& Technology \\
SPC & Website: www.sciencepubco.com/index.php/IJET \\
Research paper & . \\
\hline
\end{tabular}

\title{
Energy Efficiency in Virtualized Data Center
}

\author{
Tehmina Karamat Khan ${ }^{1 *}$, Mohsin Tanveer ${ }^{2}$, Asadullah Shah ${ }^{3}$ \\ ${ }^{1}$ International Islamic University Malaysia \\ ${ }^{2}$ National University of Science and Technology Pakistan \\ ${ }^{3}$ International Islamic University Malaysia \\ *Corresponding author E-mail:asadullah@iium.edu.my
}

\begin{abstract}
Industrial and academic communities have been trying to get more computational power out of their investments. Data centers have recently received huge attention due to its increased business value and achievable scalability on public/private clouds. Infra-structure and applications of modern data center is being virtualized to achieve energy efficient operation on servers. Despite of data center advantages on performance, there is a tradeoff between power and performance especially with cloud data centers. Today, these cloud applicationbased organizations are facing many energy related challenges. In this paper, through survey it has been analyzed how virtualization and networking related challenges affects energy efficiency of data center with suggested optimization strategies.
\end{abstract}

\section{Introduction}

With the proliferation of cloud computing, large scale data centers with hundreds of blade server nodes are consuming significant energy resources. Number of organizations implementing data center architecture has been increased at record breaking rate in attendance [1]. Many practices can be tested to achieve maximum energy efficiency such as energy efficient hardware resources, application algorithms, DVFS (Dynamic Voltage and Frequency Scaling) and virtualization of hardware resources. Through virtualization one can create many Virtual Machines (VMs) on one physical server. Applications running on multiple densely packed servers can be combined through virtualization. Virtual Machines assist software and hardware heterogeneity because of its ability to move from one physical host to another. With the expeditious development of VMware, KVM and XEN technologies for virtualization, one can achieve benefits such as easy server management, reduction in cost, efficient resource utilization, location independency, dynamic resource adjustment and elimination of redundancy. Although virtualization can save up to $20 \%$ energy on minimum nodes but at the same time server virtualization can degrade its maximum throughput due to hypervisors. In depth analysis of data center server energy usage, the following possible overheads are deduced [2].

- Server consolidation: when the server is idle, a sufficient power is utilized by the servers. Energy cost can be reduced by applying strategy.

- Multicore optimization: For multi-threaded applications multicore optimization mechanism can reduce the energy utilization.

- Virtual machines: As the virtualized servers runs on more energy as compare to physical servers. Optimal number of virtual machine can decrease the energy cost.

- Hypervisor: Energy consumption can greatly be reduced, if hypervisor architecture be further analysed.

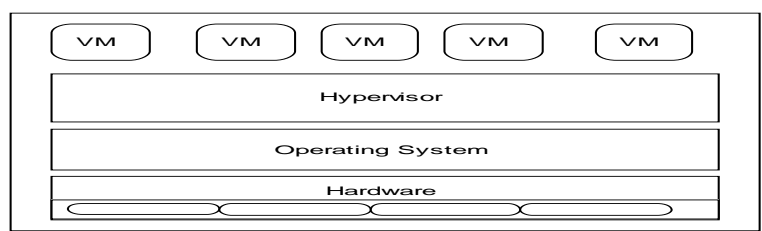

Fig. 1: Principle of Virtualization

This paper will explain potential performance overheads of server consolidation, optimization strategies based on surveys and virtualization effects on energy efficiency. Networking related overheads will also be discussed in this paper. The rest of this paper is outlined as follow. Section II, will explain the background and related work. Section III, will discuss the models of virtualization. Section IV, will discuss the architecture. Section V, will highlight Networking overheads. The last section will be conclusion and future work.

\section{Related work}

1975 [13] is the first research which discusses the application of virtualization as in concept of time sharing and multiprogramming. Queuing network model or zero queues is the most recent study with estimation of high performance prediction. However this model hasn't been validated properly with any specific VM architecture. Cost reduction with different workload scenarios has been discussed in [3] by proposing an architecture for energy efficient resource utilization and algorithms scheduling. An empirical analysis of Xen and KVM has been conducted in [4] focusing on virtual machine scalability and performance. [5] Proposed architecture of energy management for VDC by dividing resources into local power management strategies and global policies. In [6]Y. Song discussed the solution for multi-tier applications, according to proposal resource will be allocated to multi applications with priorities. In [7] an object oriented framework for modular resource management has been discussed in detail. Data center dynamic thermal resource efficiency has been discussed thoroughly 
in [8], the study proposed how energy can be saved with temperature aware workload with thermal management software.

\section{Virtualization}

\subsection{Virtualization overview}

Virtualization allows you to run multiple isolated environments on the same machine as shown in figure 2. The Virtual Machine Manager (VMM) also refers to Hypervisors and both OS (gues OS) as in figure runs concurrently on top of VMM. The purpose of VMM is to virtualize all resources and allocate these resources to multiple Virtual Machines. VMM schedules the Virtual Machines and controls the access to resources. Thus VMM runs in a supervisor mode and Virtual Machines runs in a user mode.

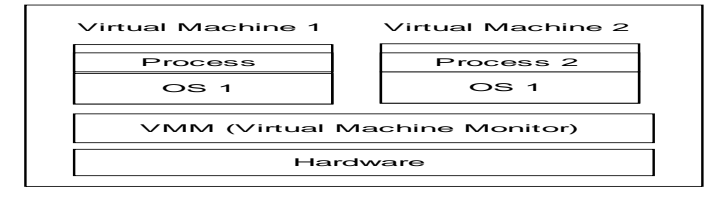

Fig.2: Virtualization

Hypervisors on the basis of hardware interaction can be classified into two types. Xen belongs to type 1 hypervisor. Xen is an open source VMM, which uses a privilege domain 0 to modify guests. On the other hand KVM(Kernel-based Virtual Machine) belongs to type 2 hypervisor which runs directly on actual physical hardware as shown in Figure 3.

\subsection{CPU virtualization}

Xen allows the user to pick and configure different CPU schedulers. Till last year's three different CPU schedulers have been introduced. Borrowed Virtual Time (BVT), Simple Earliest Deadline First (SEDF) and Credit Scheduler [11]. The default CPU scheduler is a Credit based scheduler.

BVT is based on virtual time, sending off the runnable virtual machine with smallest virtual time first. BVT is pre-emptive, Work Conserving -mode only, optimally fair and has low overhead implementation on multiprocessors. The lack of NWC mode led to need of the next scheduler.

SEDF uses real time algorithms to make sure of deliveries. With the introduction of WC mode in SEDF, the level of details present in the definition of period impacts the fairness of scheduler. SEDF is pre-emptive, Work Conserving and NWC mode, fair (depending on value of period) and implements /CPU queue (lacking global load balancing).

Credit Scheduler features auto load balancing of virtual CPU, guaranteeing no Virtual-CPU idles during runnable working period. Credit scheduler is pre-emptive, Work Conserving and NWC mode as well as supports global load balancing on multiprocessors.

KVM (kernel based virtual machine) has default Linux CPU scheduler. KVM is different from other solutions which can utilize Linux kernel component such as CFS - Completely Fair Scheduler to treat all guests as normal.

Xen and KVM, despite of their architectural difference, these VMMs are allocating and balancing global load on multi processors.

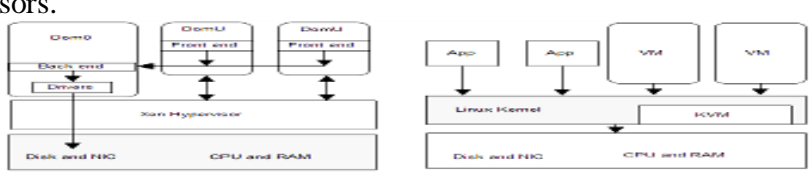

Fig. 3: Xen and KVM architectural difference

\subsection{Virtualized networks}

Xen and KVM both provide the following networking mechanisms:
- Shared Bridged Connection (shared physical Network device) - xenbr0 in XEN; br0 in KVM.

- Virtual Firewall Router (VFL)

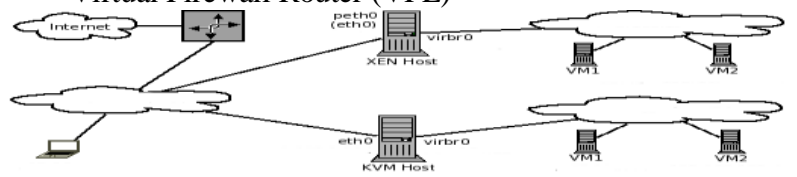

Fig. 4: Virtualized Network

In contrast, KVM virtual network bridging and routing uses network TAP. This difference lead to more energy consumption by KVM when they do network oriented tasks [12].

From Table 1, It has been observed that Xen hypervisor consumes less energy than KVM for various tasks, although KVM is easy to configure on host OS in linux. KVM networking itself consumes almost 1/3rd of more energy than Xen Hypervisor [2].

\subsection{Comparison table}

Table 1: The comparison between Xen and KVM. Performance based attributes

\begin{tabular}{|l|l|l|}
\hline & XEN & KVM \\
\hline CPU Performance & high & high \\
\hline Throughput(i/o) & medium & medium \\
\hline Resource control & Some on hypervisor & $\begin{array}{l}\text { Mostly on hypervi- } \\
\text { sor + OS }\end{array}$ \\
\hline Observance from guest & $\begin{array}{l}\text {-Resource usage } \\
\text {-Hypervisor statis- } \\
\text { tics }\end{array}$ & $\begin{array}{l}\text {-Resource usage } \\
\text {-Hypervisor statistics } \\
\text {-OS inspection }\end{array}$ \\
\hline OS guests & Yes, can be different & Yes, can be different \\
\hline Request Per second & High (3530) & High (3223) \\
\hline Memory allocation & $\begin{array}{l}\text { possible double- } \\
\text { caching }\end{array}$ & $\begin{array}{l}\text { possible double- } \\
\text { caching }\end{array}$ \\
\hline Hypervisor Complexity & $\begin{array}{l}\text { high (complex hy- } \\
\text { pervisor) }\end{array}$ & medium \\
\hline CPU Allocation & VCPU limit & VCPU limit \\
\hline $\begin{array}{l}\text { Idle server overhead } \\
\text { (energy) }\end{array}$ & $1 \%$ & $10 \%$ \\
\hline $\begin{array}{l}\text { Networking Overhead } \\
\text { Range }\end{array}$ & $2.8-70.2(\%)$ & $59.6-271.3(\%)$ \\
\hline
\end{tabular}

\section{Architecture analysis and conclusion}

In this proposed architecture figure 5 , there are $\mathrm{N}$ to $\mathrm{N}+1$ physical node. Virtualization layer run on these nodes to abstract networking resources, storage, memory and processors. Each node may contain multiple processors or cores. Furthermore, virtualization layer provides resources to Virtual Machines. But due to VM consolidation, there is a probability of not getting requested level of resources.

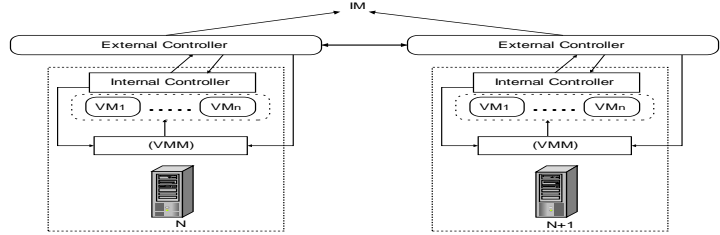

Fig. 5: System architecture

Software Architecture is comprised of external and internal controller and an integration manager.

External controller receives information from internal controller, on the basis of which external controller issue commands for resizing VM, re scheduling and migration to VMM. External controller is responsible for turning off idle nodes. External controller would also perform optimization strategies for distribution of resources. Each external controller is attached to 1 other node to send and get response from other external controllers and internal controllers. The purpose of decentralization is to avoid affects caused by Node Failures. In this case integration manager will handles failure nodes. Internal controller looks after the resources, 
node usage and thermal state of that node. Overall, Internal controller sends node states to External controllers.

\subsection{Strategy}

The migration of VMs has to be performed on the following basis:

- If resource utilization is high reaching upper threshold near to $99 \%$, VMs distribution would take place between capable nodes.

- If resource utilization is low and below the lower threshold, all VMs would have to migrate leaving that node idle. Idle node must be turned off then.

\section{Network communication}

The cost of networking for data centers is mainly concentrated onto load balancers, switches and routers, peering and inter-data centers. Some other cost contributions are based on regional facilities like WAN (traffic vol. and fibre miles). These costs can be reduced by applying clever transit strategies and with optimal placement of micro and mega data centers.

Multiple apps run inside a single data center with its own hosted app (Virtual Machine). Data center network contains two types of network traffic [13]. (a) Traffic route between external ends and internal servers. (b) Traffic route between internal servers.

An application is associated with one or more routable public IP addresses to support external requests from internet for clients. Inside the data center, processing of requests is done by front-end servers. The request spreading to front end servers is done by load balancers.

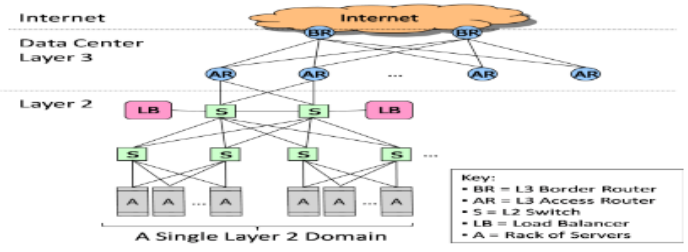

Fig.6: Network architecture for Data centers (adapted from Cisco) [14]

As shown in figure 6, layer 2 size is limited to 4000 servers in practice. VLAN is configured on Layer 2 dividing up into subnets (1 subnet per VLAN). The following problem disturbs the efficiency with this traditional approach:

- Under-utilization of resources

Load balancing technique such as half NAT and direct server return requires that all (Direct IP addresses) in a virtual IP addresses be in the same layer [15]. This means application can use available servers in the same layer if the application requires more front-end servers. On the other hand, full NAT allows server to spread across the same layer but limiting the visibility of client IP.

- Poor server connectivity

The bandwidth capacity between servers can be quite limited. This problem could be viewed as global optimization problem.

\subsection{Strategy}

- Location independent addresses should be used within services. This will allow servers to become a part of any server pool.

- If the available bandwidth is location-independent then services can be distributed between servers. The network must ensure that services are isolated from other services in data centers.

\section{Future and Conclusions}

This paper reported a survey analysis on recently published papers on Data Center related Challenges. It is concluded that Virtualiza- tion itself consumes much greater energy than physical nodes itself. It has been discussed that how energy consumption can be reduced through many ways, by ideal hypervisor selection, System architecture strategies, switching off idle nodes, changing networking configurations. Altogether there is a fundamental trade off due to hypervisors occupation of system resources for its execution. In future scope, algorithm for proposed architectures will be discussed. Furthermore, auto energy aware data center reallocation policies will be proposed on the basis of optimized architecture over multiple nodes.

\section{References}

[1] R. Brown, et al., Report to congress on server and data center energy efficiency: Public law 109-431. Lawrence Berkeley National Lab, 2008.

[2] Energy Efficiency and Server Virtualization in Data Centers: An Empirical Investigation Yichao Jin and Yonggang Wen Divison of Computer Communications School of Computer Engineering Nanyang Technological University.

[3] A. Beloglazov et al., Energy-aware resource allocation heuristics for efficient management of data centers for cloud computing. Future Generation Computer Systems, Elsevier, May, 2011.

[4] T. Deshane, et al., Quantitative comparison of Xen and KVM. Xen Summit, Boston, MA, USA, pp. 1-2, Jun, 2008.

[5] R. Nathuji and K. Schwan. Virtualpower: Coordinated power management in virtualized enterprise systems. ACM SIGOPS Operating Systems Review, 41(6):265-278, 2007.

[6] Y. Song, H. Wang, Y. Li, B. Feng, and Y. Sun. Multi-Tiered On-Demand resource scheduling for VM-Based data center. In Proceedings of the 2009 9th IEEE/ACM International Symposium on Cluster Computing and the Grid-Volume 00, pages 148-155, 2009.

[7] C. Waldspurger and W. Weihl. An Object-Oriented Frame-work for Modular Resource Management, In Proc. of the 5th International Workshop on Object Orientation in Operating Systems, pages 138143, October 1996

[8] R. K. Sharma, C. E. Bash, C. D. Patel, R. J. Friedrich, and J. S. Chase. Balance of power: Dynamic thermal management for internet data centers. IEEE Internet Computing, pages 42-49, 2005.

[9] Y. Bard. Performance Analysis of Virtual Memory Time-Sharing Systems. Proc.of IBM Systems Journal, 14(4):366-384, 1975.

[10] P. Barham, et al., Xen and the Art of Virtualization. In Proc. of the 19th ACM symposium on operating systems principles, Dec, 2003.

[11] L. Cherkasova et al., Comparison of the three CPU schedulers in Xen. Sigmetrics Performance Evaluation Review 35(2): pp. 42-51, 2007.

[12] Yichao Jin, Yonggang Wen and Qinghua Chen, "Energy Efficiency and Server Virtualization in Data Centers: An Empirical Investigation," IEEE INFOCOM Workshop on Communications and Control for Sustainable Energy Systems: Green Networking and Smart Grids, pp. 133-138,Mar. 2012.

[13] The Cost of a Cloud:Research Problems in Data Center Networks Albert Greenberg, James Hamilton, David A. Maltz, Parveen Patel Microsoft Research, Redmond, WA, USA.

[14] Ahmed, Syed Faiz, et al. "Model Predictive Controller-based, Single Phase Pulse Width Modulation (PWM) Inverter for UPS Systems." ACTA POLYTECHNICA HUNGARICA 11.6 (2014): 23 38.

[15] E. R. Hinden. Virtual router redundancy protocol (VRRP). RFC $3768,2004$. 